\title{
Lusisersaly
}

\section{Electrical characterization of radio frequency discharges}

Mahony, CMO., Maguire, PD., \& Graham, WG. (2005). Electrical characterization of radio frequency discharges. Plasma Sources Science and Technology, 14(2), S60-S67. https://doi.org/10.1088/0963-0252/14/2/S08

Link to publication record in Ulster University Research Portal

\section{Published in:}

Plasma Sources Science and Technology

Publication Status:

Published (in print/issue): 01/05/2005

DOI:

10.1088/0963-0252/14/2/S08

\section{Document Version}

Publisher's PDF, also known as Version of record

\section{General rights}

Copyright for the publications made accessible via Ulster University's Research Portal is retained by the author(s) and / or other copyright owners and it is a condition of accessing these publications that users recognise and abide by the legal requirements associated with these rights.

\section{Take down policy}

The Research Portal is Ulster University's institutional repository that provides access to Ulster's research outputs. Every effort has been made to ensure that content in the Research Portal does not infringe any person's rights, or applicable UK laws. If you discover content in the Research Portal that you believe breaches copyright or violates any law, please contact pure-support@ulster.ac.uk. 


\title{
Electrical characterization of radio frequency discharges
}

\author{
C M O Mahony ${ }^{1}$, P D Maguire ${ }^{1}$ and W G Graham ${ }^{2}$ \\ ${ }^{1}$ Nanotechnology Research Institute, N.I. Bio-Engineering Centre, University of Ulster, \\ BT37 0QB, UK \\ ${ }^{2}$ Department of Physics and Astronomy, Queen's University Belfast, BT7 1NN, UK
}

Received 5 December 2004, in final form 9 March 2005

Published 4 May 2005

Online at stacks.iop.org/PSST/14/S60

\begin{abstract}
Two electrical techniques that are frequently used to characterize radio frequency plasmas are described: current-voltage probes for plasma power input and compensated Langmuir probes for electron energy probability functions and other parameters. The following examples of the use of these techniques, sometimes in conjunction with other diagnostic methods, are presented: plasma source standardization, plasma system comparison, power efficiency, plasma modelling and complex processing plasma mechanisms.
\end{abstract}

\section{Introduction}

The fabrication of many future micro- and nano-technology devices will depend on plasma processing techniques. Characterization of the plasma condition is becoming critical as process scaling progresses.

There are many possible diagnostic methods employed in plasma characterization, including electrical, particle flux, optical, microwave, radio and modelling techniques, of which current-voltage $(I-V)$ probes and Langmuir probes are the most common. This is in part due to the relative ease of set-up, the wide range of internal plasma parameters that can be surveyed and, for $I-V$ probes, the potential for noninvasive real-time plasma monitoring and control. There are, however, a number of significant issues regarding the accuracy of measurement and subsequent parameter extraction. These issues are influenced by both the choice of gas mixture and also the radio frequency (rf) source configuration.

Here, we present the details of experimental techniques and results of parameter extraction for both capacitively (CCPs) and inductively coupled plasmas (ICPs) and for a number of model gases, namely hydrogen and argon. We also show how characterization can assist the understanding of a number of plasma and plasma-surface processes in the more complex argon-chlorine $(\mathrm{Ar} / \mathrm{Cl})$ processing environment, which contains negative ions and reactive radicals.

The next two sections summarize the set-up and methodology for $I-V$ probe and compensated Langmuir probe use, illustrated by our own measurements in both the $13.56 \mathrm{MHz}$ rf driven UK GEC CCP Reference Cell and also the $13.56 \mathrm{MHz} \mathrm{Ar} / \mathrm{Cl} \mathrm{ICP}$. In the final section, examples are shown of the use of these characterization techniques for the standardization of rf plasma sources $[1,2]$, to provide input parameters for plasma modelling [3,4], to allow comparison of data between differing plasma systems and groups of investigators by, for example, power efficiency measurements [5], and to clarify the behaviour of complex processing plasmas $[6,7]$.

\section{2. $I-V$ probes}

\subsection{Parameters}

The electrical characterization of $\mathrm{rf}$ sources involves the accurate measurement of $\mathrm{rf}$ current and voltage and their respective phase difference at the $\mathrm{CCP}$ or ICP sample electrode [1,2] or at the ICP coil [5,8-11]. These measurements can be used to determine plasma impedance, both mean and instantaneous power $[1,12]$, and source efficiency [5,10-12]. During the GEC Reference Cell programme $[1,2], I-V$ measurements were used to ensure electrical equivalence and thus plasma standardization at different test facilities.

\section{2. $I-V$ probe types}

$I-V$ probes are deployed as an attachment to the rf power input feed, and can be designed to be only minimally intrusive. The use of both proprietary $[1,5,13]$ and custom built derivative [12] current and voltage probes has been reported. The custom built derivative probe used by the authors (figure 1) consists of an inductive current pick up and a capacitive voltage probe close to the powered electrode (or ICP coil). 


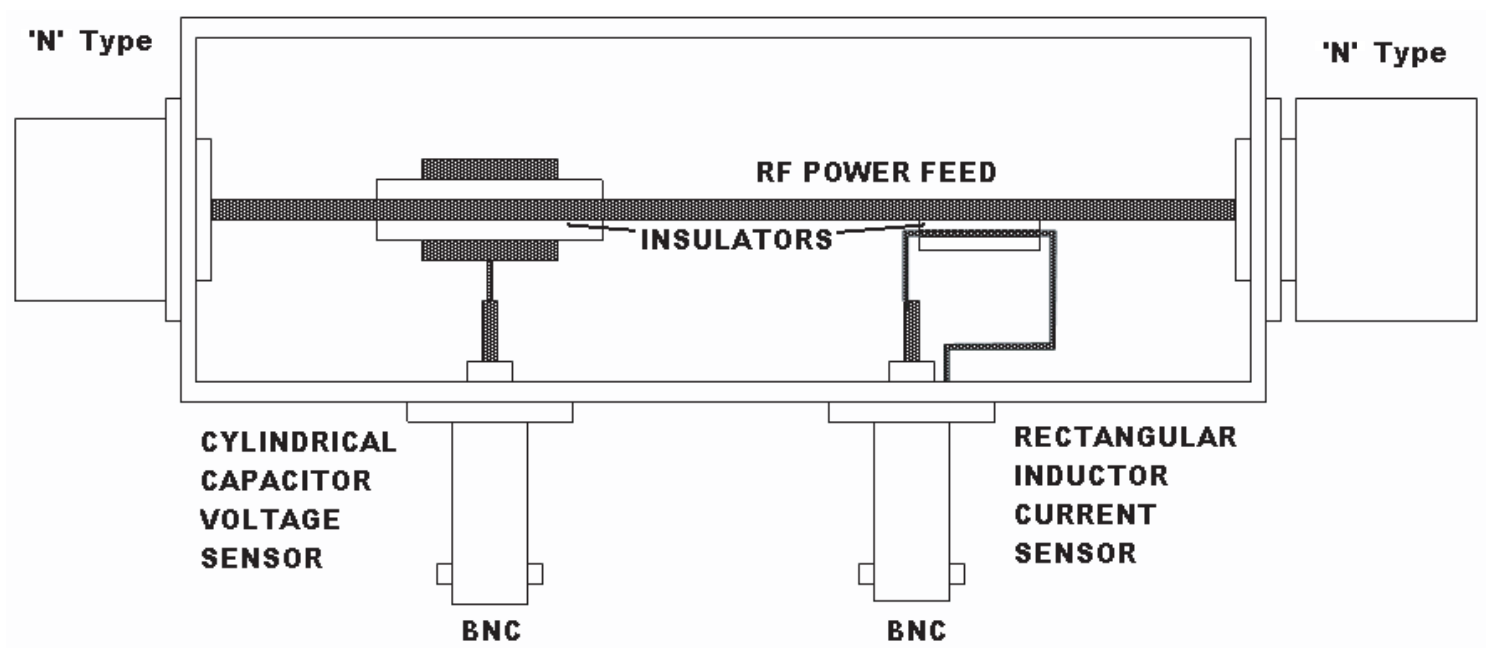

Figure 1. In line derivative $I-V$ probe used in the UK GEC CCP Reference Cell by the authors.

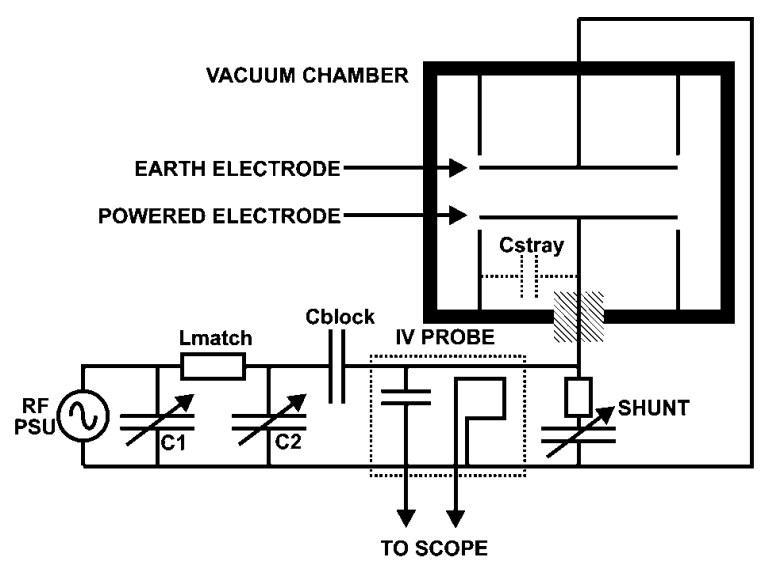

Figure 2. Schematic diagram of $I-V$ measurement circuit for the UK GEC CCP Reference Cell showing rf generator, matching network (C1, C2 and Lmatch), blocking capacitor (Cblock), $I-V$ probe and shunt.

\subsection{I-V data analysis}

To determine the current and voltage at the electrode or coil, an equivalent electrical circuit model is solved $[1,2]$. This model accounts for the remote positioning of the $I-V$ probes and stray external circuit impedances that can be measured with the plasma off.

The major issue in the use of $I-V$ probes is inaccuracy in $I-V$ phase difference measurements. This is due to stray impedances that allow significant parasitic currents to flow in the plasma source structure and measurement circuit.

Parasitic currents in CCPs can be almost an order of magnitude higher than the plasma currents [1], but may be reduced by the incorporation of a shunt (figure 2) to null the effect of stray capacitances [2].

In ICPs, a number of issues make $I-V$ measurement more problematic than for CCPs $[8,9,11]$. The high coil currents require the matching unit to be close to the coil to minimize resistive losses, making probe positioning difficult. Also, in ICPs, the stray impedances, particularly inductance, cause parasitic currents to circulate in the source structure.

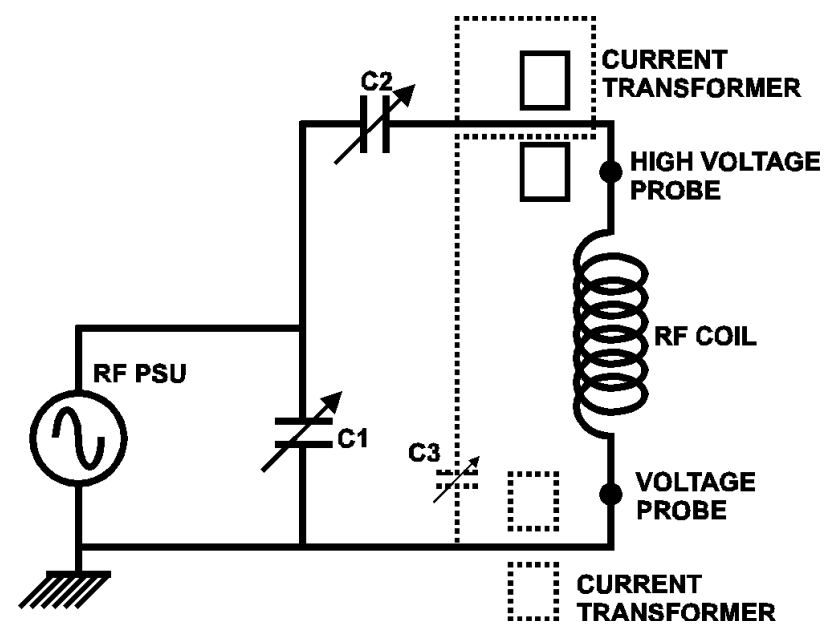

Figure 3. Schematic diagram of the coil current measurement circuit of an ICP, showing the rf generator, matching network (C1 and $\mathrm{C} 2$ ), current transformers, rf coil and voltage probes. The suggested capacitive shunt [14] is shown as the dotted connection from the high voltage line, passing in the reverse sense through the high voltage current transformer, through the variable capacitor $\mathrm{C} 3$, to the earth line. The suggested current transformer on the earthed line is shown dotted.

A schematic diagram of a typical measurement set-up is shown in figure 3.

For a long time there was no equivalent to the shunt technique employed in CCPs to reduce these currents. A recent suggestion [14], however, proposes the use of a shunt and the use of a second current transformer at the earthed end of the ICP coil (the proposed shunt and current transformer are shown as dotted in figure 3 ). The shunt across the rf coil passes in the reverse sense through the high voltage current transformer; capacitance C3 is varied when no plasma is present until equal currents are measured on both transformers. Note that the current transformer on the earthed line would also give a good approximation of coil losses. The difference between the two, when the plasma is present may give an indication of the capacitive currents in the plasma.

To develop a measurement circuit model, rf coil and support structure impedances, particularly resistance, need 
to be measured with no plasma present. Note that the mutual inductance between the plasma and the source structure depends on the plasma impedance and geometry; this relationship is difficult to assess.

Advances have been made, however; one group of investigators [5] have used a system consisting of pairs of proprietary current and voltage probes to measure $I-V$ at the input and output of the ICP matching network and a calorimetric system to account for coil power losses. Other workers [10] have recently applied the technique to a pulsed ICP. The plasma-structure mutual inductance issue, however, remains to be addressed.

\section{Compensated Langmuir probes}

\subsection{Parameters}

Langmuir probes are employed to measure electron energy probability functions (EEPFs) and other plasma parameters $\left(n_{\mathrm{e}}, T_{\mathrm{e}}, T_{\mathrm{eff}}, V_{\mathrm{p}}, V_{\mathrm{f}}, n^{+}\right)$. Electron energy distribution functions (EEDFs), which can be readily derived from measured EEPFs, are key to the characterization of rf plasmas, aiding the understanding of physical and chemical behaviour. Simple rf plasmas, e.g. argon, can be almost completely characterized by their EEDFs $[15,16]$ and accurate EEDF evaluation is needed to calculate species rate constants for models of non-Maxwellian plasmas [3,4]. As pointed out by Gagne and Cantin [17], uncompensated Langmuir probes are unreliable because of plasma potential fluctuation effects in rf plasmas. Compensated probes are regularly applied $[12,18-25]$ to CCP systems, and ICP systems with no Faraday shield, to reduce the effects of the rf modulation of plasma potential.

\subsection{Compensated Langmuir probe design}

In-house constructed, passively compensated Langmuir probes used by the authors [26, 27] (figure 4) are typically constructed from a $0.04 \mathrm{~mm}$ diameter tungsten wire extending $10 \mathrm{~mm}$ from a re-entrant hole in a supporting alumina tube. The probe body contains two pairs of self-resonant inductors to block the rf fundamental and second harmonic $(27.12 \mathrm{MHz})$. High frequency impedance is further increased by the incorporation of a low pass filter with a cut-off frequency near $500 \mathrm{kHz}$. This compensation typically reduced the rf ripple on the probe to a few millivolts. The probe body diameter is particularly thin, only $4.5 \mathrm{~mm}$ (achieved by using the smallest electrical components readily available), and diminishes plasma perturbation by the probe.

\subsection{Data analysis}

A Smartsoft $[28,29]$ automated probe control and data capture system, generates a Langmuir probe current-voltage (LPIV) characteristic, plasma parameters and EEPFs derived from the second derivative of the LPIV characteristic [30]. The system includes an automatic cleaning procedure (allowing in situ cleaning of the probe tip by electron or ion bombardment) and a reference probe to account for changes in $V_{\mathrm{p}}$ caused by the bias applied to the probe during measurement.

EEDFs in rf plasmas are rarely simple Maxwellians and hence the EEDF cannot be adequately described in terms of a single temperature, and an effective temperature, $T_{\text {eff }}$, is sometimes employed [3,31]. To better characterize these plasmas, a post-analysis method to fit EEDFs to single or double Maxwellian, Druyvesteyn-like, or combinations of these distributions has been developed and used (figure 5) [19,32]. A second advantage of the fitting technique is that it can be used to compensate for an underestimate of electron density near zero energy.

Note that the Druyvesteyn analysis method [30], which assumes the EEPF is proportional to $\mathrm{d}^{2} I / \mathrm{d} V^{2}$ for a cylindrical probe, cannot adequately measure EEPFs at low electron energies. The usual Druyvesteyn zero second derivative definition of the plasma potential implies that the EEPF at zero electron energy, $\operatorname{EEPF}(0)=0$, whereas for any real distribution, EEPF $(0)>0$. This apparent contradiction is a result of initial assumptions made by Druyvesteyn, and limits

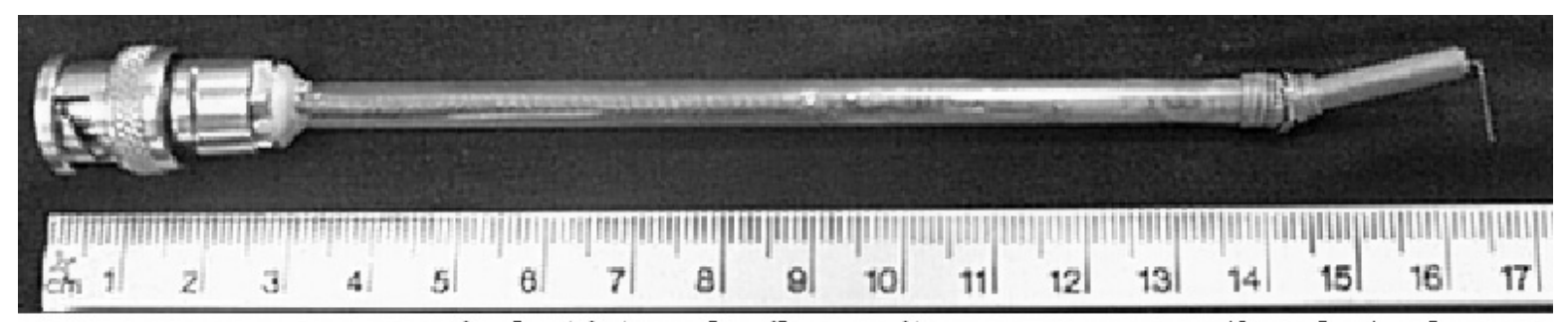

equivalent internal coil capacitances compensation electrode

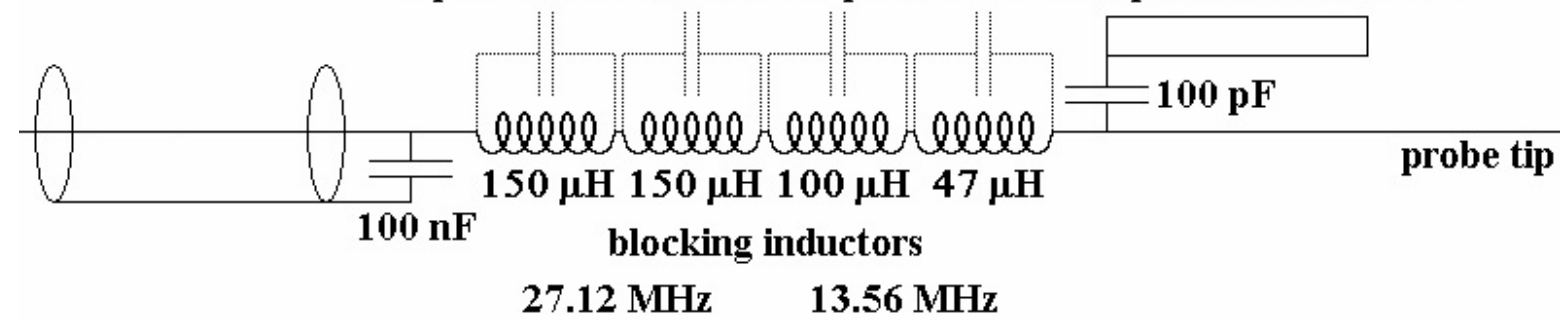

Figure 4. Photograph, schematic and circuit diagram of the compensated Langmuir probe used in the UK GEC CCP Reference Cell and an $\mathrm{Ar} / \mathrm{Cl}$ ICP. The blocking inductors occupy the region from the 9 to $13 \mathrm{~cm}$ marks on the ruler. 


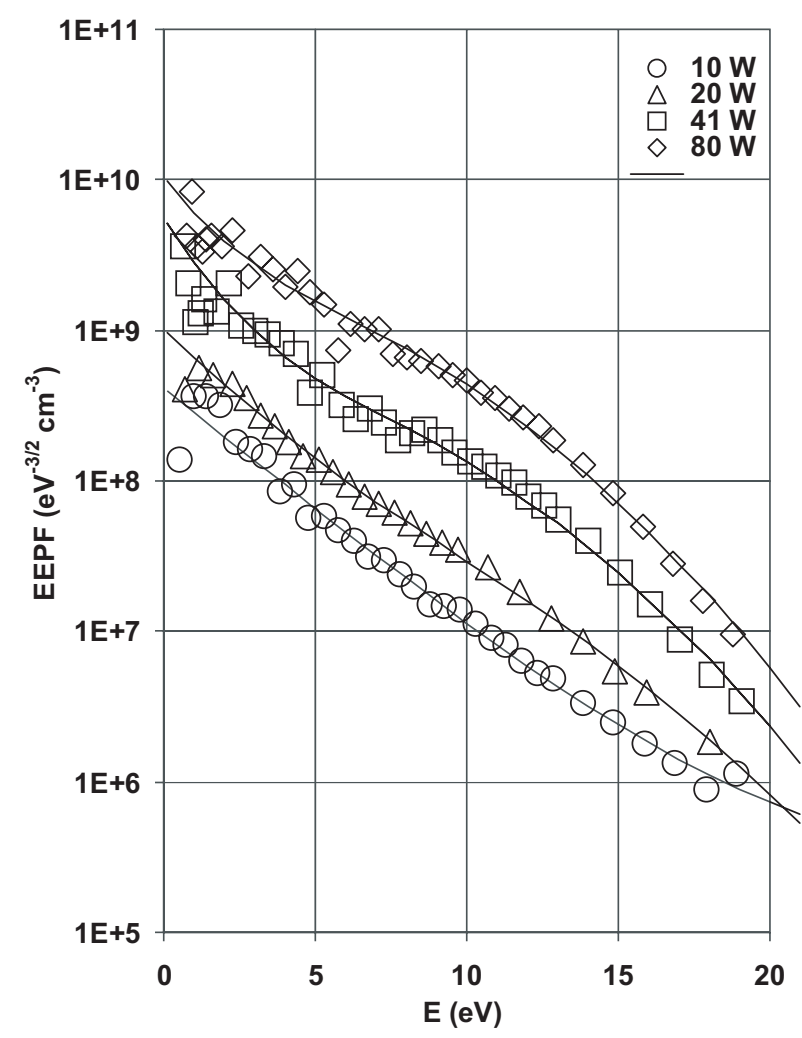

Figure 5. Raw argon EEPFs (symbols) from the $\mathrm{Ar} / \mathrm{Cl}$ ICP and EEPFs fitted using the analysis programme (lines). Powers are measured at the rf generator.

the applicability of that analysis. Additional issues concern the measurement of $n^{+}$, particularly in the case where negative ions may be present; see, for example, $[25,33,34]$ and references therein.

\subsection{Compensated Langmuir probe procedures}

The use of compensated Langmuir probes is problematic since there are many potential sources of error, particularly in rf plasmas [33, 35].

Valid application of the Druyvesteyn formula requires collisionless electron motion near the probe; i.e. the Debye length $\lambda_{\mathrm{D}}$ must be much less than the electron mean free path $\lambda_{\mathrm{e}}$. The probe and probe holder radii ( $a$ and $b$, respectively) must also be much less than $\lambda_{\mathrm{e}}$ to be effectively non-intrusive. These conditions were fulfilled for our probes and for the plasma conditions we have investigated; i.e. $a=40 \mu \mathrm{m}$, $b=2.25 \mathrm{~mm}$, the longest value of $\lambda_{\mathrm{D}}$ was $\sim 0.6 \mathrm{~mm}$ while the shortest value of $\lambda_{\mathrm{e}}$ was $\sim 5 \mathrm{~mm}$. The probe tip was mounted so that no contact was possible between it and the insulating support structure and the active area of the probe tip was bent through $90^{\circ}$ to avoid shielding of the probe tip by the support structure.

Probe contamination is reduced by continuously cleaning (with the automated procedure) by pulsed electron bombardment except during the short periods $(\sim 1 \mathrm{~s})$ required for the data capture. In addition, EEPF measurements are taken at predetermined electron energies in a random order so that probe-heating effects are minimized. Probe circuit resistance is accounted for by the use of the reference probe. $\mathrm{Rf}$ distortion is minimized by the self-resonant inductors and low pass filter, which reduce the rf ripple on the probe to a few millivolts for the fundamental and orders of magnitude less for the second through to the fifth harmonic. The DC resistance of the blocking inductors and filter is typically $20 \Omega$, and is accounted for in the SmartSoft acquisition system.

The effects of drift in the plasma parameters is reduced by the experimental procedure; a number of near-identical $I-V$ characteristics are required before an EEPF measurement is carried out; the EEPF measurements are then repeated to check for drift before being considered valid. Note that EEPF measurements and LPIV characteristics are the result of significant averaging.

Accurate measurement of the ion current and the valid subtraction of its extrapolated value from the probe current to determine the EEPF is fraught with potential errors [35]. For the small diameter probes used here, an ion correction method based on Laframboise theory [36], modified by a Bohm correction factor of 1.6, is incorporated in the software. For light gases, Godyak et al [35], state that the maximum error in EEPFs determined by these methods is less than a factor of two for electron energies below $9 T_{\mathrm{e}}$. For the heavier gases used here, and from our measured $T_{\mathrm{e}}$ values (ranging from 2 to $4 \mathrm{eV}$ ), we estimate our EEPF, and thus EEDF, measurements to be valid up to $\sim 20 \mathrm{eV}$.

Comparison of EEPF measurements in rf plasmas is difficult because power measurement methods and differences in plasma chamber geometries do not allow accurate assessment of discharge conditions. Typically, quoted rf powers are measured at the rf generator; no account of losses in the external circuitry or parasitics is considered. True system power efficiencies vary considerably and can be as low as $10 \%$. However, if the conditions outlined above have been followed, comparison of EEPF measurements between groups can give some insight into power efficiency and specific chamber geometrical factors.

\section{Other characterization techniques}

It is beyond the scope of this short work to consider in detail all of the electrical characterization methods that are available. Examples are: the use of ion flux probes $[37,38]$ and mass-energy analysers [39] to measure ion/neutral fluxes and energies. New techniques are emerging: the use of rf frequency analysis for plasma tool characterization [40] and recent developments in hairpin resonators [41] (for the local measurement of $n_{\mathrm{e}}$ ), show promising results. It is worth noting other characterization methods are often used in conjunction with electrical techniques; these include laser induced fluorescence (LIF), photodetachment, microwave methods and emission and absorption spectroscopy $[3,6,7]$.

\section{Application of characterization}

\subsection{CCP standardization}

$I-V$ measurement of current and voltage has been successfully used to ensure electrical equivalence between GEC Reference Cell CCPs in different test facilities and allowed the accurate determination of deposited plasma power $[1,2,12]$. The initial 


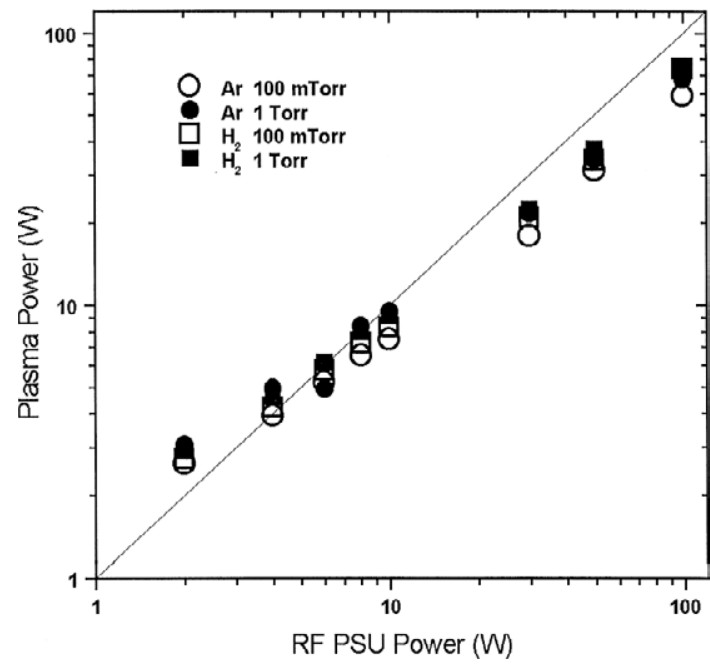

Figure 6. Derivative probes and shunt measurements of rms rf plasma power versus rf power measured at the rf generator in the UK GEC CCP Reference Cell.

CCP GEC reference cell standardization study [2] compared six different cells with different power supplies, matching elements and current and voltage measuring devices. The measured voltage and current amplitudes at the fundamental frequency agreed to within about $4 \%$ and $14 \%$, respectively, and phase difference to within $4 \%$. Values of plasma power had spreads of up to $25 \%$. The electrical characteristics of the UK GEC CCP Reference Cell fall well within that spread [12]. The study [2] showed that the electrical characteristics of the discharges are strongly affected by the external circuit elements particularly the type and make of rf generator and matching elements. In fact, the length of connecting cables and their layout can influence the harmonic content. Figure 6 shows typical deposited plasma power measurements made in the UK GEC CCP Reference Cell, indicating source efficiencies of $\sim 70 \%$ for rf input powers greater than $20 \mathrm{~W}$ in argon and in hydrogen.

\subsection{Time resolved power measurements}

The synthesis of two techniques, fast ICCD imaging of plasma excitation and instantaneous rf power measurements, has been used to elucidate heating mechanisms in a CCP $[18,42,43]$. Time resolved emission data were obtained by imaging the central plasma onto the fast-gated ICCD. The measured emission signal was deconvoluted with the radiative lifetime of the excited state to derive excitation images across the $25 \mathrm{~mm}$ electrode gap shown in the upper part of figure 7. The time variation of plasma power determined using derivative probes for the same conditions is shown in the lower portion. There is clearly good phase correlation between the observed excitation and power input to the bulk $(\sim 30 \mathrm{~ns})$ and near electrode $(\sim 15 \mathrm{~ns})$ excitation. Note that the negative excursions in instantaneous power, e.g. at $\sim 70 \mathrm{~ns}$, are believed to be genuine, and show that the plasma drives energy into the rf supply system at this phase of the cycle.
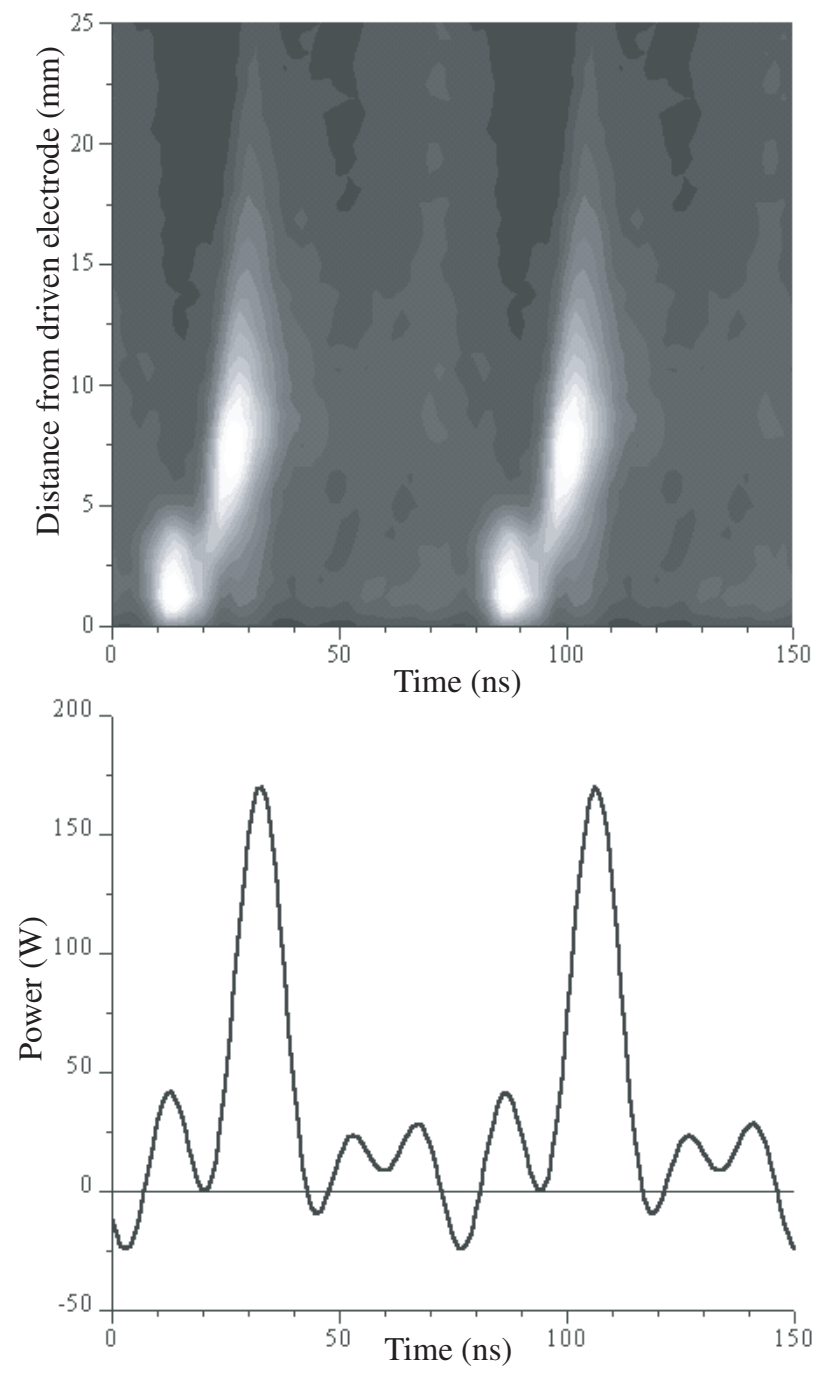

Figure 7. Excitation of the hydrogen $656.3 \mathrm{~nm}$ (Balmer $\alpha$ ) line in the UK GEC CCP Reference Cell (upper) and the associated instantaneous rf plasma power. Pressure $=250 \mathrm{mTorr}$, plasma power $=35 \mathrm{~W}$.

\subsection{ICP Source power efficiency}

Despite the difficulty of using $I-V$ probes in ICPs, measurements have been made and indicate deposited plasma power efficiencies of between $10 \%$ and $90 \%$ [5, 10,11]. The most likely causes of this spread in efficiencies are differences in rf coil, Faraday shield and chamber geometries and measurement inaccuracies. The most credible ICP plasma power measurements [5] can be used in conjunction with an extensive data set of argon EEPFs from the same workers [31] to aid comparison of ICP behaviour between different groups and between experiment and theory. EEPFs are better indicators of plasma behaviour than $n_{\mathrm{e}}$ values; plasmas with significantly different EEDFs, and thus plasma properties, may have the same electron density.

In figure 8,10 mTorr argon EEPFs measured in the $\mathrm{Ar} / \mathrm{Cl}$ ICP using procedures outlined in section 3, and equivalent measurements by Godyak and Kolobov [31] can be seen to have very similar shapes, especially above $5 \mathrm{eV}$. Note that at the highest powers, EEPF values measured below $\sim 2.5 \mathrm{eV}$ in 


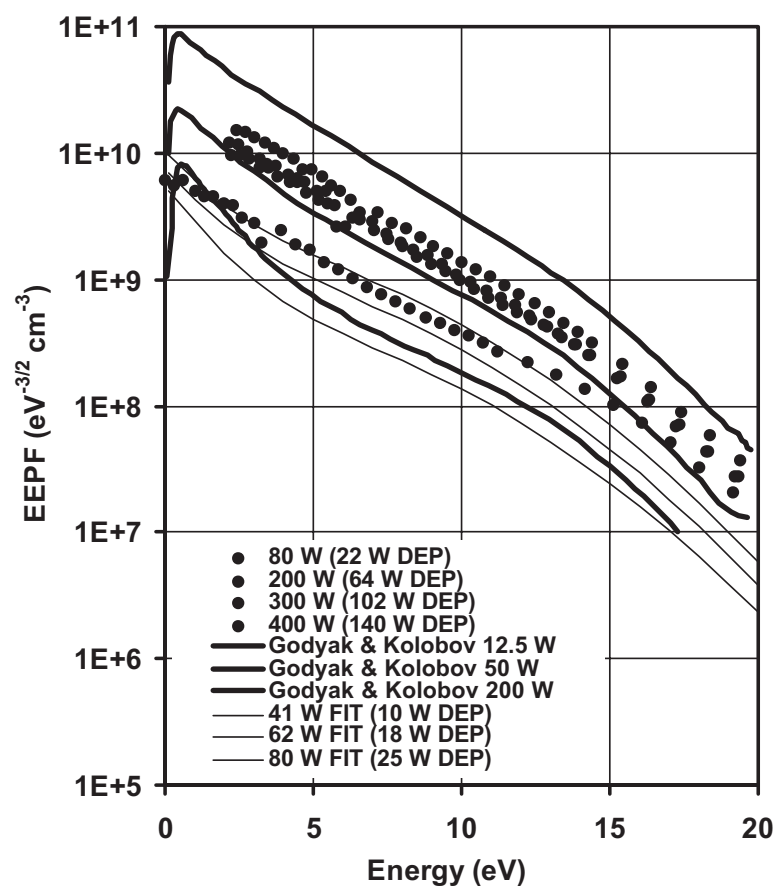

Figure 8. Comparison of different 10 mTorr argon EEPFs. Heavy lines are from Godyak and Kolobov [31] for three deposited plasma powers. Thin lines are fits to the author's $\mathrm{Ar} / \mathrm{Cl}$ ICP low power data. Black circles are the author's higher power data points. The $\mathrm{Ar} / \mathrm{Cl}$ ICP powers are measured at the rf generator and the estimated deposited plasma powers are labelled 'DEP'.

the $\mathrm{Ar} / \mathrm{Cl} \mathrm{ICP}$ were not consistent because the high currents drawn near $V_{\mathrm{p}}$ caused significant probe heating.

There are major geometrical differences between the two ICPs. Godyak and co-worker's [5,31] ICP is purpose built for experimentation and has a relatively small rf coil diameter $(127 \mathrm{~mm})$ whereas the $\mathrm{Ar} / \mathrm{Cl} \mathrm{ICP}$ has more similarities with a process chamber. In the $\mathrm{Ar} / \mathrm{Cl} \mathrm{ICP}$, the separation between the rf coil and its conducting support is about $10 \mathrm{~mm}$, whereas for the Godyak and co-workers [5,31] ICP it is greater than $60 \mathrm{~mm}$. It is reasonable to assume that the power efficiency of the $\mathrm{Ar} / \mathrm{Cl}$ ICP system is significantly lower because of the larger stray currents induced in the ICP structure. In addition, Godyak and co-workers [5,31] use a Faraday shield to limit capacitive mode coupling whereas the $\mathrm{Ar} / \mathrm{Cl} \mathrm{ICP}$ does not.

Direct comparison of these EEPFs allows deposited powers, shown in brackets in the legend of figure 8 , to be estimated. The $10 \mathrm{~m}$ Torr ICP power coupling efficiencies presented by Godyak and co-workers [5] increase from about $50 \%$ at $12.5 \mathrm{~W}$ deposited power to saturate at about $80 \%$ for deposited powers above $100 \mathrm{~W}$. The measured efficiencies in the author's $\mathrm{Ar} / \mathrm{Cl} \mathrm{ICP}$ system exhibit the same trend with increasing deposited power; however, these values were consistently about half those of Godyak and co-workers [5].

\subsection{Modelling data}

In plasma processing models, EEDFs are frequently assumed to be pure Maxwellians. This leads to inaccuracies in the calculation of electron-heavy-particle reaction rates when nonMaxwellian EEDFs pertain. EEDFs derived from measured EEPFs can be used to reduce these errors.
A zero-dimensional kinetic model, which used the approach of Bassett and Economou [44] as a starting point, has been employed to calculate species densities in the $\mathrm{Ar} / \mathrm{Cl}$ ICP. Rate coefficients for electron-heavy-particle reactions were calculated using the experimentally derived EEDFs [3, 4]. Other rate coefficients were found in the literature $[44,45]$. Thirty potential plasma reactions and eighteen potential wall interactions were considered and plasma neutrality was assumed. Estimation of wall losses included the assumption of a modified Bohm criterion for positive ions; the sheath edge plasma density was decreased with respect to the bulk plasma density by a 'Bohm factor' $\left(f_{\mathrm{B}}<1\right)$. The nine particle balance equations were reduced to three simultaneous equations with three unknowns $\left(\mathrm{Cl}_{2}\right.$ density, $\mathrm{Cl}^{-}$density and $\left.f_{\mathrm{B}}\right)$, which were solved iteratively. The resultant Bohm factor varied between 0.08 and 0.2 depending on process conditions. The species densities and fluxes were then used to compare wall losses from a modified ambipolar diffusion model for three species (positive ions, negative ions and electrons) and good agreement was obtained.

Wall interactions were accounted for by using literature values of wall recombination rates for the formation of molecular chlorine from atomic chlorine [44,45] and by assuming sticking coefficients of 0.0001 for both atomic and molecular chlorine. The fragmentation ratio for the two channels:

$$
\mathrm{Cl}_{2}^{+} \rightarrow \mathrm{Cl}_{2}+\mathrm{e}
$$

and

$$
\mathrm{Cl}_{2}^{+} \rightarrow 2 \mathrm{Cl}+\mathrm{e}
$$

was estimated to be $a: b=1: 99$.

Atomic chlorine densities predicted by the kinetic model can be compared (figure 9) with atomic chlorine densities measured in the $\mathrm{Ar} / \mathrm{Cl} \mathrm{ICP}$ by 2-photon LIF using xenon as a calibration gas. Note that the literature-derived $\mathrm{Xe}$ cross-section value [46] resulted in impossibly large measured dissociation fractions (above 100\%). An empirical scaling factor for the cross-section was thus introduced based solely on the variation of cross-section with radius for other inert gases. The use of this empirical factor gave plausible dissociation trends and values over the range of source pressures and rf powers investigated. These measurements of $\mathrm{Cl}$ density are the first of their kind and comparison with the literature is, therefore, not possible. The $\mathrm{Cl}$ densities so determined are in close agreement with the values predicted by the model (figure 9). Recently, the chlorine atomic density measurements have been repeated in the $\mathrm{Ar} / \mathrm{Cl}$ ICP under the same experimental conditions but using $\mathrm{CCl}_{4}$ as the calibration gas [47]; absolute density measurements are in very good agreement with the values reported in figure 9 .

Improvements to the zero-dimensional model, for example, the use of the measured plasma power to provide additional production and loss balance information will be presented in the near future, and extension of the code to a one-dimensional model is under way.

\subsection{Clarification of processing behaviour}

We have applied these electrical characterization techniques, in conjunction with optical measurements and zero-dimensional 

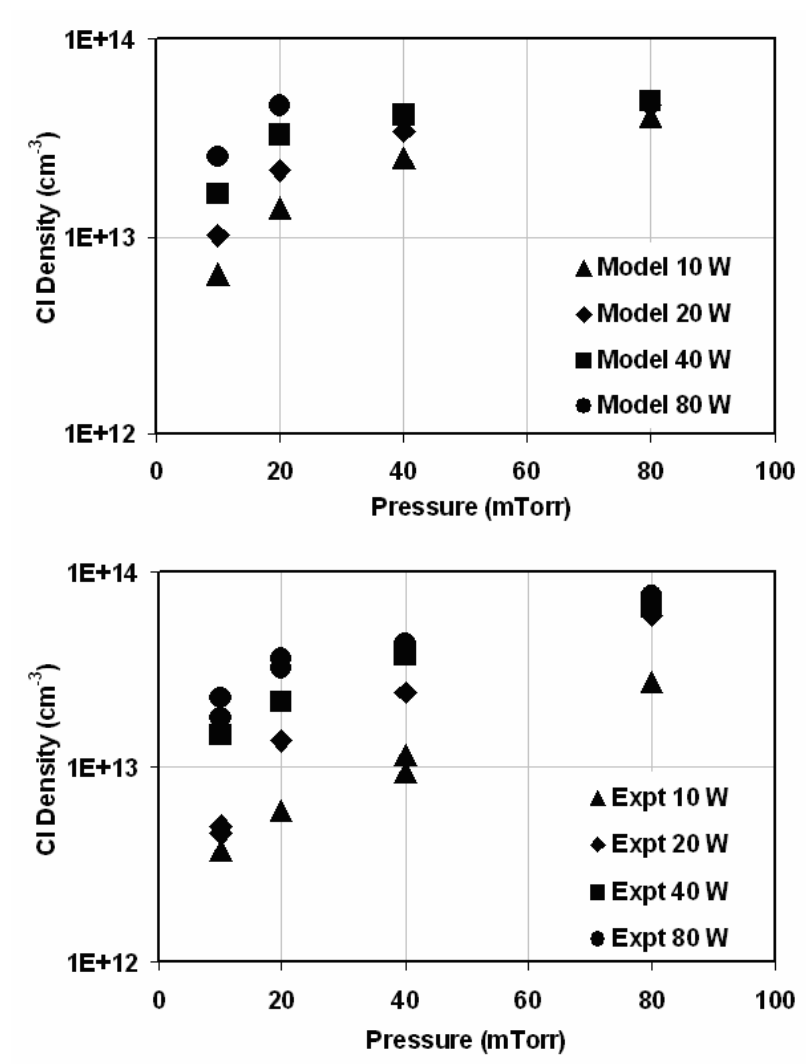

Figure 9. ICP characterization of a $5 \% \mathrm{Cl}_{2}, 95 \%$ Ar mixture for a range of $\mathrm{rf}$ generator powers and pressures: $\mathrm{Cl}$ atomic density predicted by the zero-dimensional kinetic model (upper) and measured in the Ar/Cl ICP by LIF (lower).

kinetic modelling, to a range of different systems, and to a number of argon-chlorine ICP based plasma processes. The presence of highly reactive atomic chlorine in these plasmas limits the range of applicability of many characterization techniques. Cavity ring down mirrors, employed to measure $\mathrm{Cl}^{-}$via photodetachment, suffered from rapid reduction in reflectivity. Langmuir probe contamination is also problematic; however, the combination of the in situ probe cleaning technique, manual cleaning and the use of standard conditions to validate probe operation, allowed an extensive EEPF data set to be recorded.

Measurements of species densities by LIF and probebased photo-detachment, augmented by the zero-dimensional kinetic model using measured EEPFs and EEDFs, allow sample substrate fluxes to be estimated. In this way, the properties of plasma-processed samples were linked to processing system controls via the species fluxes, allowing optimum processing conditions to be determined.

Surface modification of silver to produce $\mathrm{Ag} / \mathrm{AgCl}$ matrices is used to reduce the electrode-electrolyte interface impedance of electrochemical and biological sensors [6]. New microscale electrode systems based on thin film silver cannot be exposed to traditional wet electrochemical techniques. We have, therefore, investigated the use of low power (less than $40 \mathrm{~W}$ ) $\mathrm{Ar} / \mathrm{Cl}$ plasma induced $\mathrm{AgCl}$ growth on $\mathrm{Ag}$ thin films. The $\mathrm{Ag}_{x} \mathrm{Cl}_{y}$ stoichiometry and resultant impedance and electrode stability was found to be dependent on an optimal $\mathrm{Cl}$ atom surface flux, along with low energy surface ion bombardment. The latter appears to enhance neutral atomic $\mathrm{Cl}$ reactions with the substrate, to produce higher bond strengths. From the plasma characterization and model, the key bombardment species was probably $\mathrm{Cl}^{+}$with a possible contribution from $\mathrm{Ar}^{+}$, while $\mathrm{Cl}_{2}^{+}$was thought to play a much less significant role. As the power is increased, the higher energy ion bombardment leads to an etch reaction competing with growth.

Plasma etching of gallium nitride [7] for photonic device fabrication and switching/transmission applications was investigated in $\mathrm{Ar} / \mathrm{Cl}$ at high powers $(>400 \mathrm{~W})$ and high substrate biases. Diagnostics have highlighted a number of different etch mechanisms across the various parameter ranges. This (along with zero-dimensional modelling) is central to optimizing the etch process in terms of rate, etched surface stoichiometry, roughness and selectivity. High $\mathrm{GaN}$ etch rates, with minimal adverse stoichiometry and roughness, have been achieved in the $\mathrm{Ar} / \mathrm{Cl} \mathrm{ICP}$ at significantly reduced $\mathrm{Cl}_{2}$ fractions. As the halogen component of the gas mixture is increased, the $\mathrm{Cl}$ atom $/ \mathrm{Cl}^{+}$ion ratio increases (with a significant reduction in ion flux) and optimal etching was observed once this ratio reaches a threshold. More detailed measurements of species flux at the sample electrode are in progress and with input from the modelling, this will enhance our understanding of the plasma and plasma-surface interactions, for low-energy surface modification and high-energy etch processes.

\section{Summary}

The electrical characterization of rf plasmas can be problematic because of a number of issues: these include rf coupling and matching, plasma potential modulation, harmonic content, phase measurement, stray impedances and differing plasma source construction. Here, the implementation and use of two important electrical plasma characterization techniques, namely $I-V$ probes and Langmuir probes, have been described.

$I-V$ probes can be used to measure the complex current and voltage at rf plasma source electrodes (or coils). A shunt is often employed to increase the accuracy of phase measurements by nulling stray impedances, thereby reducing parasitic currents in the source and measurement circuit $[1,2,12,14]$. The resulting measurements, combined with an electrical circuit model which accounts for probe position and stray impedances, not only generate electrode (or coil) currents and voltages but also plasma impedance, plasma power (mean and instantaneous) and source efficiency.

The effects of the modulated plasma potential can be successfully compensated for in Langmuir probe measurements. When combined with careful probe, probe circuit design and measurement procedures [33,35], these probes can be used to measure a wide range of plasma parameters, such as charged particle densities and the plasma potential. Importantly, they can also be used to determine the EEDF, highlighting their often non-Maxwellian nature. These accurately measured EEDFs are essential in calculations of plasma generated species densities and fluxes.

The use of these techniques in source standardization, comparison of measurements made on differing plasma systems and by differing investigators, power efficiency 
calculation, plasma modelling and behaviour in a processing environment has been illustrated.

\section{References}

[1] Sobolewski M A 1995 J. Res. Natl Inst. Stand. Technol. 100341

[2] Hargis P J Jr et al 1994 Rev. Sci. Instrum. 65140

[3] Mahony C M O, Escoffier C N, Maguire P D, Corr C S, Gomez S, Costa I, Bricha E and Graham W G 2001 Bull. Am. Phys. Soc. 4638

[4] Kiehlbauch M W and Graves D B 2002 J. Appl. Phys. 913539

[5] Godyak V A, Piejak R B and Alexandrovich B M 1999 J. Appl. Phys. 85703

[6] Escoffier C, Maguire P D, Mahony C M O, Graham W G, McAdams E T and McLaughlin J A 2002 J. Electrochem. Soc. 149 H98

[7] Rizvi S A, Maguire P D, Mahony C M O, Okpalugo O A, Corr C S, Graham W G and Morley S M 2002 Phys. Status Solidi 0112

[8] Okpalugo O A, Mahony C M O, Graham W G, Laverty S and Maguire P D 2000 Bull. Am. Phys. Soc. 4524

[9] Okpalugo O A, Mahony C M O, Laverty S J, Maguire P D, Gomez S and Graham W G 2001 Bull. Am. Phys. Soc. 4621

[10] Guo W and DeJoseph C A Jr 2001 Plasma Sources Sci. Technol. 1043

[11] Miller P A, Hebner G A, Greenberg K E, Pochan P D and Aragon B P 1995 J. Res. Natl Inst. Stand. Technol. 100427

[12] Graham W G, Mahony C M O and Steen P G 2000 Vacuum 563

[13] http://www.scisys.com/hdocs/smartpim_broc.htm

[14] Piejak R B 2004 private communication

[15] Hopkins M B 1995 J. Res. Natl Inst. Stand. Technol. 100415

[16] Godyak V A, Piejak R B and Alexandrovich B M 2002 Plasma Sources Sci. Technol. 11525

[17] Gagne R R J and Cantin A 1972 J. Appl. Phys. 432639

[18] Mahony C M O, McFarland J, Steen P G and Graham W G 1999 Appl. Phys. Lett. 75331

[19] Toader E I, Graham W G, Mahony C M O and Steen P G 2002 Rev. Sci. Instrum. 732974

[20] Overzet L J and Hopkins M B 1993 J. Appl. Phys. 744323

[21] Singh H and Graves D B 2000 J. Appl. Phys. 874098
[22] Gudmundsson J T 1998 Plasma Sources Sci. Technol. 7330

[23] Wendt A E 2001 Rev. Sci. Instrum. 722926

[24] Braithwaite N St J, Benjamin N M P and Allen J E 1987 J. Phys. E 201046

[25] Allen J E 1995 Plasma Sources Sci. Technol. 4234

[26] Mahony C M O, McFarland J and Graham W G 1997 Proc. Frontiers II (Bad Honnef, Germany) p 179

[27] McFarland J, Mahony C M O, Steen P G and Graham W G 1997 Bull. Am. Phys. Soc. 421757

[28] http://www.scisys.com/hdocs/downloads/smartprobe.pdf

[29] Hopkins M B and Graham W G 1986 Rev. Sci. Instrum. 57 2210

[30] Druyvesteyn M J 1930 Z. Phys. 64781

[31] Godyak V A and Kolobov V I 1998 Phys. Rev. Lett. 81369

[32] Mahony C M O 2003 The development of advanced measurement techniques for the characterisation of low pressure technological processing plasmas $P h D$ Thesis University of Ulster

[33] Sudit I D and Woods R C 1994 J. Appl. Phys. 764489

[34] Franklin R N 2003 J. Phys. D: Appl. Phys. 36823

[35] Godyak V A, Piejak R B and Alexandrovich B M 1992 Plasma Sources Sci. Technol. 136

[36] Laframboise J G 1966 Institute for Aerospace Studies University of Toronto Report \#100

[37] Braithwaite N St J 1997 Plasma Sources Sci. Technol. 6133

[38] Braithwaite N St J, Booth J P and Cunge G 1996 Plasma Sources Sci. Technol. 5677

[39] Rees J A 1999 Vide 54441

[40] Law V J, Kenyon A J, Thornhill N F, Seeds A J and Batty I 2001 J. Phys. D: Appl. Phys. 342726

[41] Piejak R B, Godyak V A, Garner R, Alexandrovich B M and Sternberg N 2004 J. Appl. Phys. 953785

[42] Mahony C M O and Graham W G 1999 IEEE Trans. Plasma Sci. 2672

[43] Mahony C M O, Al Wazzan R and Graham W G 1997 Appl. Phys. Lett. 71608

[44] Bassett N L and Economou D J 1994 J. Appl. Phys. 751931

[45] Christophorou L G and Olthoff J K 1999 J. Phys. Chem. Ref. Data 28131

[46] Ono K, Oomori T and Tuda M 1992 Japan. J. Appl. Phys. 31 L269

[47] Marro F G and Graham W G 2005 private communication 